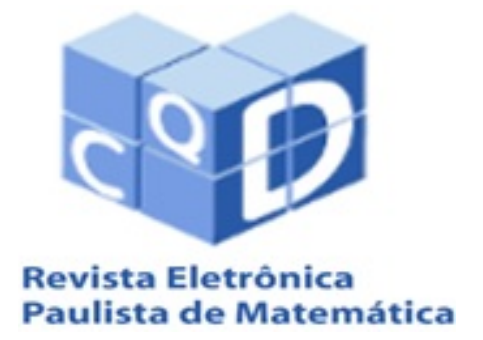

ISSN 2316-9664

Volume 14, fev. 2019

Edição Ermac

Iniciação Científica

Enayle Maryane Teixeira Paes

Universidade Federal de

Alfenas-MG

enaylepaes@gmail.com

Juliana Maria da Silva

Universidade Federal de

Alfenas-MG

juliana.silva@unifal-

mg.edu.br

\section{Estudo numérico da concentração de partículas para escoamento em tubo permeável aplicado ao processo de filtração tangencial}

Numerical study of the concentration of particles for flow in permeable tube applied to the crossflow filtration process

\section{Resumo}

O processo de filtração tangencial tem sido frequentemente estudado devido a sua ampla aplicabilidade em diversos processos industriais (indústrias alimentícias, químicas, farmacêuticas). Este trabalho apresenta um estudo numérico relacionada ao processo de filtração tangencial. O depósito de partículas ao longo da superfície permeável de um tubo, durante um escoamento, é analisado. O mecanismo de transporte de massa é matematicamente representado pela equação de convecção-difusão com associadas condições iniciais e de fronteira para coordenadas cilíndricas. A equação é discretizada utilizando-se a técnica de diferenças finitas. Comparações de dois esquemas upwinding para aproximação dos termos convectivos são apresentadas. Os efeitos de parâmetros físicos no perfil de concentração são investigados. Os resultados, mostraram-se qualitativamente e quantitativamente com boa concordância com os dados da literatura.

Palavras-chave: Simulação numérica. Diferenças Finitas. Transferência de Massa. Filtração Tangencial.

\begin{abstract}
The crossflow filtration process has often been studied due to its wide applicability in several industrial processes (food, chemical and pharmaceutical industries). This work presents a numerical study related to the crossflow filtration process. The deposition of particles along the permeable surface of a tube during a flow is analyzed. The mass transport mechanism is mathematically represented by the convection-diffusion equation with associated initial and boundary conditions for cylindrical coordinates. The equation is discretized by using the finite-difference technique. Comparisons of the two upwinding schemes for approximation of convective terms are presented. The effects of physical parameters on the concentration profile are investigated. The results were shown qualitatively and quantitatively with good agreement with the literature data.
\end{abstract}

Palavras-chave: Numerical simulation. Finite-difference. Mass transference. Crossflow filtration. 


\section{Introdução}

O processo de filtração tangencial com membranas tubulares é adotado por várias indústrias, como por exemplo, nas indústrias de alimentos para clarificação de vinhos, sucos de frutas e vinagre, na remoção de levedura de cerveja, na separação de bactérias e gorduras do leite; nas indústrias químicas, no processamento de tintas e derivados de petróleo; nas indústrias farmacêuticas e de cosméticos, para produção de água ultrapura; dentre outras.

No processo de filtração tangencial em membranas tubulares (ou tubos permeáveis), a pressão impele somente parte do produto através da membrana, enquanto que o produto restante flui tangencialmente à superfície permeável, como se pode observar na Fig. 1. A separação de partículas de materiais distintos ocorre espontaneamente em função de seus tamanhos, independentemente da temperatura e da densidade do material a separar [1,2]. Durante a filtração tangencial, partículas dentro do fluxo de alimentação são convectivamente dirigidas para a superfície da membrana onde elas acumulam, enquanto que o movimento difusivo de partículas causa um transporte, dessas partículas, oposto ao transporte convectivo do escoamento de filtrado (permeado) $[1,2]$.

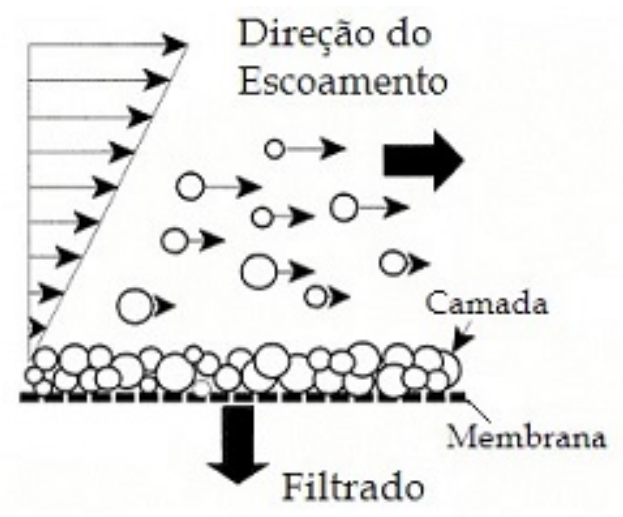

Figura 1: Esquema de filtração tangencial [1].

O acúmulo de partículas próximo à superfície da membrana é conhecido como concentração de polarização, o qual é um fenômeno que causa uma complicação adicional ao processo de filtração, pois provoca o aumento na resistência hidráulica para o fluxo de permeado e, consequentemente, o declínio do fluxo com o tempo. Por isso, um melhor conhecimento da formação da camada e a deposição de partículas na membrana poderia resultar em um uso econômico da filtração tangencial em muitas aplicações técnicas [1, 2]. Assim, uma investigação minuciosa dos fenômenos de transporte é necessária para o melhor entendimento dos mecanismos de transferência de massa presentes no processo de filtração tangencial.

Em função da importância do assunto, numerosos estudos experimentais, analíticos e numéricos, para simular escoamentos de fluidos em tubos permeáveis, têm sido assunto de intensas pesquisas nos últimos anos. Em particular, uma variedade de trabalhos dedicados à modelagem numérica para analisar a concentração de polarização em filtração tangencial é encontrada na literatura. Lee e Clark [3] investigaram o declínio do fluxo devido à camada de polarização durante a filtração tangencial e predizeram o estado estacionário do fluxo de permeado. Mignard e Glass [4] apresentaram um modelo para prever efeitos de variação do pH nos mecanismos de entupimento 
(fouling), próprios do processo de concentração de soluções de proteínas por microfiltração. Paris et al. [5] discutiram os modelos para o fluxo de permeação e propuseram modificações no modelo de resistência em série para considerar a influencia da concentração média de soluto na resistência devido à camada de polarização. Richardson e Nassehi [6] propuseram um modelo para simular o campo de concentração ao longo de uma parede permeável, para escoamento de fluidos não-newtonianos. Damak et al. [7] apresentaram uma investigação do depósito de partículas sobre a superfície permeável em membranas tubulares. Venezuela et al [8] utilizou a técnica híbrida analítico-numérica, conhecida por GITT (Generalized Integral Transform Technique), para modelagem e simulação da equação de conservação das espécies químicas, na investigação do escomento laminar incompressível, newtoniano e permamente em tubos permeáveis. Silva et al. [9] desenvolveu uma modelagem para estudo da concentração, a qual simula escoamentos de fluidos newtonianos e não-newtonianos (power-law) em tubos permeáveis, em regime laminar, usando três diferentes modelagens upwind. Diante disso, observa-se que a necessidade de um método numérico preciso e eficiente para a solução dos processos de filtração tangencial continua a estimular uma grande quantidade de pesquisas.

A fim de investigar a formação da camada de concentração próxima à superfície permeável por meio de um modelo matemático simplificado, o objetivo deste trabalho é apresentar um estudo numérico para escoamento laminar de soluções aquosas em tubos permeáveis. Para isso, a equação de convecção-difusão, a qual modela a distribuição de partículas sólidas no escoamento em tubos permeáveis (processo de filtração tangencial), será discretizada, pela a técnica de diferenças finitas, e implementada.

Em escoamentos em que a convecção é importante, a discretização adequada dos termos convectivos da equação que representa o referido escoamento é extremamente essencial para a qualidade da solução numérica. A escolha do tipo de discretização pode gerar oscilações não físicas e consequentemente acarretar instabilidade numérica, por isso, o desenvolvimento de esquemas upwind para aproximar termos convectivos (em geral não-lineares) tem sido um importante assunto nas últimas três décadas (ver, por exemplo, $[10,11,12,13])$. Portanto, uma avaliação da influência e desempenho de dois diferentes esquemas convectivos na equação de convecçãodifusão é apresentada neste pesquisa.

Esse trabalho é uma continuação do estudo apresentado no Ermac 2018 [14], com uma análise minuciosa da influência da malha computacional e parâmetros adimensionais no perfil de concentração do escoamento.

\section{Descrição do modelo numérico}

Nesta investigação é considerado um escoamento incompressível, isotérmico e completamente desenvolvido em tubo cilíndrico com paredes permeáveis.

\subsection{Formulação matemática}

A formulação matemática adimensional é determinada pela equação diferencial de convecçãodifusão, a qual representa a equação de conservação das espécies químicas [2]:

$$
u \frac{\partial c}{\partial z}+v \frac{\partial c}{\partial r}=\frac{2}{R e S c} \frac{1}{r} \frac{\partial}{\partial r}\left(r \frac{\partial c}{\partial r}\right)
$$


em que $z$ é a coordenada axial, $r$ é a coordenada radial, $u$ é a velocidade axial, $v$ é a velocidade radial, $c$ é a concentração de soluto e $R e=\frac{\rho u_{0} 2 R}{\mu}$ ( $\rho$ é a massa específica, $u_{0}$ é velocidade axial de entrada, $R$ é o raio do tubo e $\mu$ é a viscosidade dinâmica) e $S c=\frac{\mu}{\rho D}$ ( $D$ é o coeficiente de difusão) são os números adimensionais de Reynolds e Schmidt, respectivamente.

As velocidades adimensionais radial, $v$, e axial, $u$, utilizadas nesse trabalho são obtidas de YUAN [15] (apud [8])

$$
\begin{gathered}
v(r)=-\frac{2 R e_{w}}{r R e}\left[r^{2}-\frac{1}{2} r^{4}+\frac{R e_{w}}{72}\left(-4 r^{2}+9 r^{4}-6 r^{5}+r^{6}\right)+\frac{R e_{w}^{2}}{10800}\left(166 r^{2}-380 r^{4}+\right.\right. \\
\left.+275 r^{5}-75 r^{6}+15 r^{7}-r^{8}\right] \\
u(z, r)=u_{1}(r) u_{2}(z),
\end{gathered}
$$

com

$$
\begin{gathered}
u_{1}(r)=1-r^{2}+\frac{R e_{w}}{36}\left(-2+9 r^{2}-9 r^{4}+2 r^{5}\right)+\frac{R e_{w}^{2}}{10800}\left(166-760 r^{2}+825 r^{4}-300 r^{5}+75 r^{6}-6 r^{7}\right), \\
u_{2}(z)=\frac{1}{1-\frac{R e_{w}}{18}+\frac{83 R e_{w}^{2}}{5400}}+4 \frac{R e_{w}}{R e} z
\end{gathered}
$$

em que $R e_{w}=\frac{\rho v_{w} 2 R}{\mu}\left(v_{w}\right.$ é a velocidade de permeação, a qual a será considerada constante) é o número de Reynolds na parede permeável.

\subsection{Condições de fronteira}

Os limites do domínio numérico estão representados na Fig. 2. O fluxo é simétrico e, portanto, apenas metade do tubo é considerada. As condições de fronteira para velocidades e concentração de soluto na forma adimensional são definidas por $[7,16]$

( i ) entrada: um perfil laminar não desenvolvido é prescrito

$$
z=0, \quad 0 \leq r \leq 1, \quad u=1, \quad v=0, \quad c=1
$$

( ii ) eixo de simetria: não há fluxo de massa passando pela fronteira, portanto

$$
r=0, \quad 0 \leq z \leq \frac{L}{R}, \quad \frac{\partial u}{\partial r}=0, \quad v=0, \quad \frac{\partial c}{\partial r}=0,
$$

em que $L$ é o comprimento do tubo; 
( iii ) superfície permeável: a velocidade axial é nula, a velocidade radial é definida pela velocidade de permeção (constante nesse trabalho) e a concentração é definida pela equação de balanço de massa, $N_{S}=v_{w} c_{w}-D \frac{\partial c}{\partial r}$, considerando-se nulo o fluxo de soluto através do tubo permeável, pois, no estado estacionário as partículas são quase $100 \%$ rejeitadas pela superfície permeável [2]

$$
r=1, \quad 0 \leq z \leq \frac{L}{R}, \quad u=0, \quad v=v_{w}, \quad c_{w} v_{w}=\left.\frac{2}{\operatorname{ReS} c} \frac{\partial c}{\partial r}\right|_{r=1}
$$

em que $c_{w}$ é a concentração de soluto na superfície permeável;

( iv ) saída: o fluxo desenvolvido e a baixa difusividade molecular do soluto permitem a aplicação de uma condição de perfil desenvolvido

$$
z=L, \quad 0 \leq r \leq 1, \quad \frac{\partial u}{\partial z}=0, \quad \frac{\partial v}{\partial z}=0, \quad \frac{\partial c}{\partial z}=0
$$

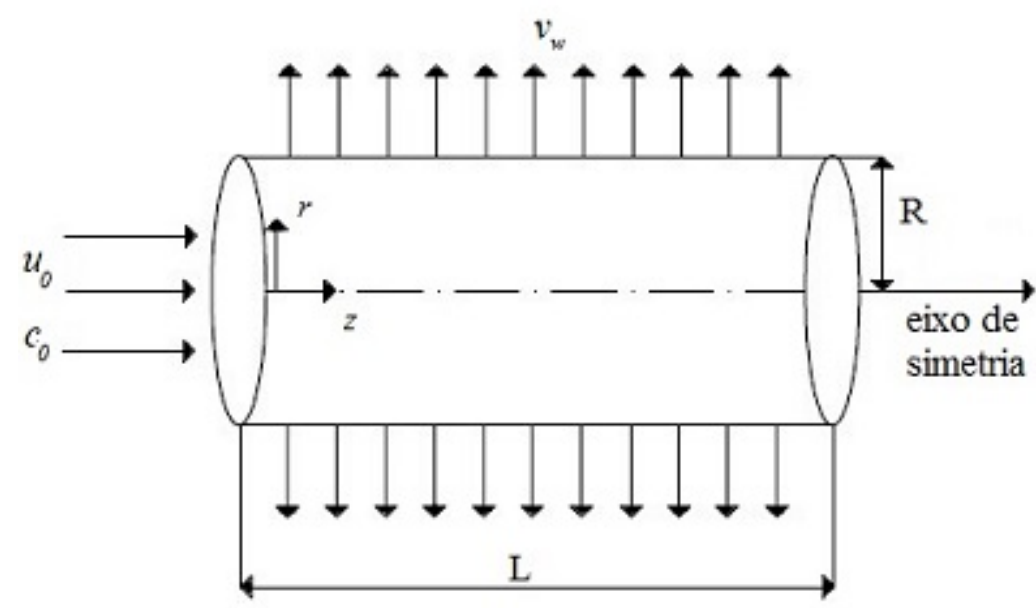

Figura 2: Domínio do problema.

\section{Método numérico}

A Eq. (1) juntamente com as condições de fronteira (6) - (9) foram discretizadas pela técnica de diferenças finitas em malha computacional co-localizada, em que todos os elementos (células) são retangulares de largura $\Delta z$ e altura $\Delta r$ e as variáveis são definidas no mesmo ponto, como mostra a Fig. 3. 


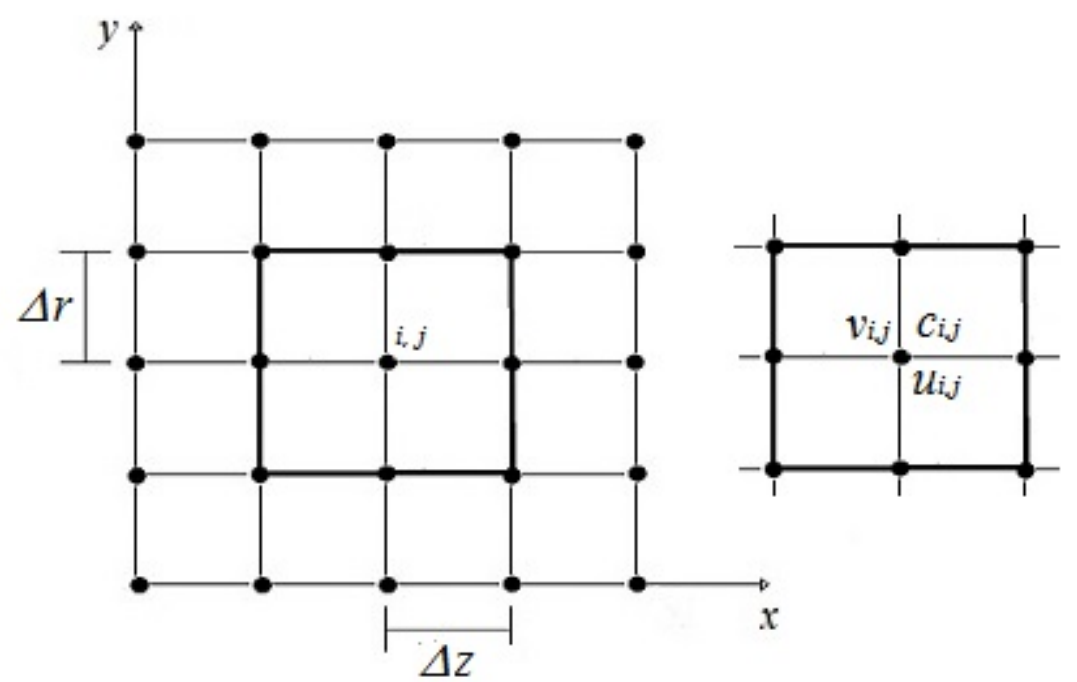

Figura 3: Malha co-localizada.

Como artifício matemático para obtenção da aproximação desejada, utilizou-se passo 1/2 nas aproximações. As derivadas do termo difusivo da Eq. (1) foram aproximadas por diferenças centrais de segunda ordem,

$$
\left.\frac{\partial}{\partial r}\left(r \frac{\partial c}{\partial r}\right)\right|_{i, j}=\frac{r_{i, j+\frac{1}{2}}\left(c_{i, j+1}-c_{i, j}\right)-r_{i, j-\frac{1}{2}}\left(c_{i, j}-c_{i, j-1}\right)}{(\Delta r)^{2}}
$$

enquanto que as derivadas dos termos convectivos foram aproximada por diferenças centrais de segunda ordem

$$
\begin{aligned}
& \left.u \frac{\partial c}{\partial z}\right|_{i, j}=u_{i, j} \frac{c_{i+\frac{1}{2}, j}-c_{i-\frac{1}{2}, j}}{\Delta z} \\
& \left.v \frac{\partial c}{\partial r}\right|_{i, j}=v_{i, j} \frac{c_{i, j+\frac{1}{2}}-c_{i, j-\frac{1}{2}}}{\Delta r}
\end{aligned}
$$

e diferenças atrasadas

$$
\begin{aligned}
& \left.u \frac{\partial c}{\partial z}\right|_{i, j}=u_{i, j} \frac{c_{i, j}-c_{i-\frac{1}{2}, j}}{\Delta z} \\
& \left.v \frac{\partial c}{\partial r}\right|_{i, j}=v_{i, j} \frac{c_{i, j}-c_{i, j-\frac{1}{2}}}{\Delta r}
\end{aligned}
$$

em que $r_{j+1 / 2}=(j+1 / 2) \Delta r$ e $r_{j-1 / 2}=(j-1 / 2) \Delta r$.

Observa-se na discretização dos termos convectivos o aparecimento dos termos $c_{i \pm \frac{1}{2}, j}$ e $c_{i, j \pm \frac{1}{2}}$, os quais não estão definidos na malha computacional, portanto, para finalizar a discretização e definir uma modelagem simplificada, esses termos foram aproximados por dois esquemas convectivos básicos, upwind de primeira e segunda ordem [12], aplicados nas Eqs. (11) - (12) e (13) - (14), respectivamente. Esses esquemas são definidos pelas seguintes relações [12]:

\section{upwind de primeira ordem}

PAES, E. M. T.; SILVA, J. M. da. Estudo numérico da concentração de partículas para escoamento em tubo permeável aplicado ao processo de filtração tangencial. C.Q.D.- Revista Eletrônica Paulista de Matemática, Bauru, v. 14, p. 30-40, fev. 2019. Edição Ermac Iniciação Científica.

DOI: 10.21167/cqdvol14ermacic201923169664emtpjms3040 Disponível em: https://www.fc.unesp.br/\#!/departamentos/matematica/revista-cqd/ 


$$
\begin{aligned}
& c_{i+\frac{1}{2}, j}=\left\{\begin{array}{rr}
c_{i, j}, & u_{i, j} \geq 0 \\
c_{i+1, j}, & u_{i, j}<0
\end{array}, \quad c_{i-\frac{1}{2}, j}=\left\{\begin{array}{rr}
c_{i-1, j}, & u_{i, j} \geq 0 \\
c_{i, j}, & u_{i, j}<0
\end{array}\right.\right. \\
& c_{i, j+\frac{1}{2}}=\left\{\begin{array}{rr}
c_{i, j}, & v_{i, j} \geq 0 \\
c_{i, j+1}, & v_{i, j}<0
\end{array}, \quad c_{i, j-\frac{1}{2}}=\left\{\begin{array}{rr}
c_{i, j-1}, & v_{i, j} \geq 0 \\
c_{i, j}, & v_{i, j}<0
\end{array}\right.\right.
\end{aligned}
$$

\section{upwind de segunda ordem}

$$
c_{i-\frac{1}{2}, j}=\left\{\begin{array}{cc}
\frac{3}{2} c_{i-1, j}-\frac{1}{2} c_{i-2, j}, & u_{i, j} \geq 0 \\
\frac{3}{2} c_{i, j}-\frac{1}{2} c_{i+1, j}, & u_{i, j}<0
\end{array} \quad, \quad c_{i, j-\frac{1}{2}}=\left\{\begin{array}{cc}
\frac{3}{2} c_{i, j-1}-\frac{1}{2} c_{i, j-2}, & v_{i, j} \geq 0 \\
\frac{3}{2} c_{i, j}-\frac{1}{2} c_{i, j+1}, & v_{i, j}<0
\end{array}\right.\right.
$$

A discretização da Eq. (1) resultou em um sistema de equações lineares, o qual foi resolvido pelo método iterativo de Gauss-Seidel [17].

\section{Resultados e discussões}

Essa seção está dividida em três partes. Na primeira parte resultados numéricos são comparados com resultados analíticos-numéricos da literatura [8]. A segunda parte envolve a definição de uma malha computacional. Por fim, na terceira parte, apresenta-se uma verificação dos resultados numéricos da modelagem com o problema físico estudado.

\subsection{Verificação dos resultados numéricos}

Com o objetivo de verificar a influência da discretização dos termos convectivos nos resultados numéricos da modelagem, comparou-se os resultados numéricos para os dois esquemas implementados neste trabalho com resultados analíticos-numéricos obtidos da literatura [8]. Para isso, foram realizadas simulações numéricas com os esquemas convectivos para $R e=300$, $D=7 \times 10^{-10} \mathrm{~m}^{2} / \mathrm{s} ; v_{w}=1,361 \times 10^{-6} \mathrm{~m} / \mathrm{s}$ e uma malha computacional de $1500 \times 600$ pontos.

A Fig. 4 apresenta uma comparação dos resultados numéricos com resultados da literatura para o perfil de concentração próximo à superfície permeável em função da coordenada axial. Observa-se que os resultados numéricos para upwind de segunda ordem apresentaram uma boa concordância com os resultados da literatura, tendo um erro relativo (ER\%) máximo de $2 \%$, enquanto que os resultados númericos para upwind de primeira ordem apresentam um maior desvio, sendo seu ER\% máximo de 10,7\% . Isto é explicado devido as limitações do equacionamento de upwind de primeira ordem, que faz com que seja introduzido maior difusão numérica aos resultados. Assim, o esquema convectivo upwind de segunda ordem será adotado nas próximas simulações. 


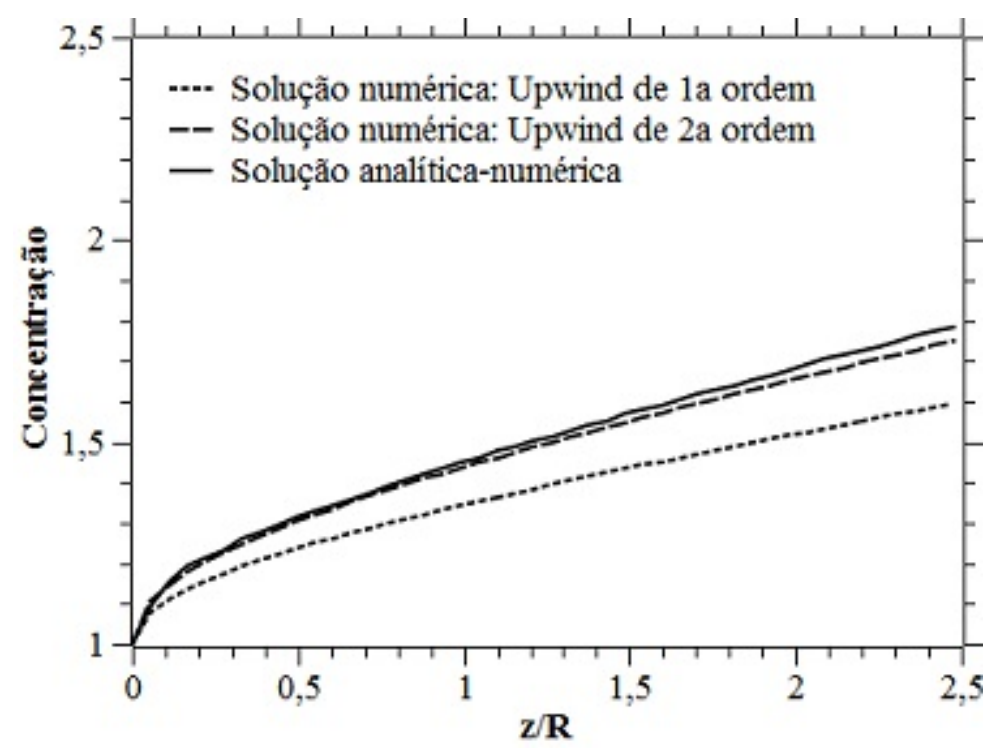

Figura 4: Comparação do perfil de concentração próximo à superfície permeável em função da coordenada axial com os resultados obtidos da literatura [8].

\subsection{Malha computacional}

Com o propósito de definir uma malha computacional refinada, porém, com baixo custo computacional, várias simulações numéricas para diferentes malhas computacionais e com diferentes números de Reynolds e Schmidt foram realizada. A Fig. 5 apresenta os perfis de concentração, $c / c_{0}$, próximo à superfície permeável em função da coordenada axial, $z / R$, para diferentes malhas computacionais. Para estes testes foram utilizados valores próximos aos encontrados na literatura [7, 8, 9]: $R e=500 ; S c=500 ; v_{w}=1,6 \times 10^{-6} \mathrm{~m} / \mathrm{s} ; R=0,003 \mathrm{~m} ; L=0,01 \mathrm{~m} ; \rho=1000 \mathrm{kgm}^{-3}$.

Pode-se notar que a partir da malha computacional $1667 \times 501$ os perfis de concentração não sofrem grandes influências, ou seja, não é necessário maior refinamento. Assim, este tamanho de malha foi adotado para os próximos testes.

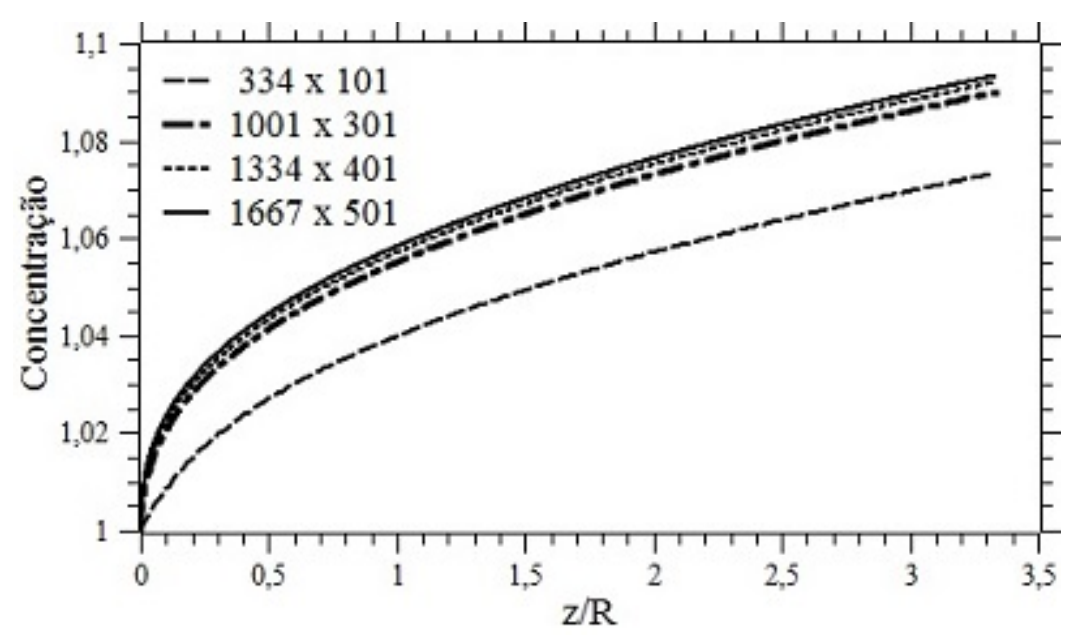

Figura 5: Concentração próxima à superfície permeável em função da coordenada axial, para diferentes malhas computacionais. 


\subsection{Variação dos números adimensionais}

Apresenta-se na Fig. 6 os perfis de concentração próximos à superfície permeável em função da coordenada axial para diferentes números de Reynolds.

Observa-se que ao aumentar o número de Reynolds a concentração ao longo do tubo diminui. Isto é explicado pelo fato que maiores números de Reynolds, tem-se maiores velocidades axiais, fazendo-se com que o arraste das partículas seja maior, diminuindo-se, assim, sua concentração próxima a camada permeável. Este mecanismo tem sido frequentemente observado no processo de filtração tangencial $[2,7,9]$, logo, os resultados numéricos da Fig. 6 estão em boa concordância com este comportamento físico.

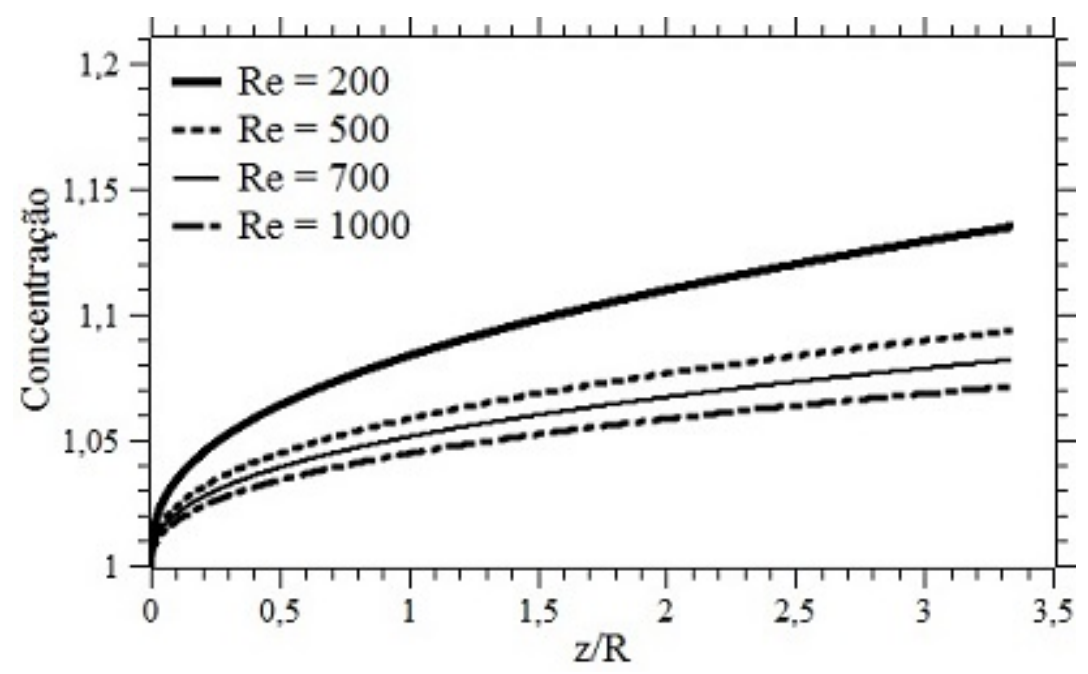

Figura 6: Concentração próxima à superfície permeável em função da coordenada axial, para diferentes número de Re.

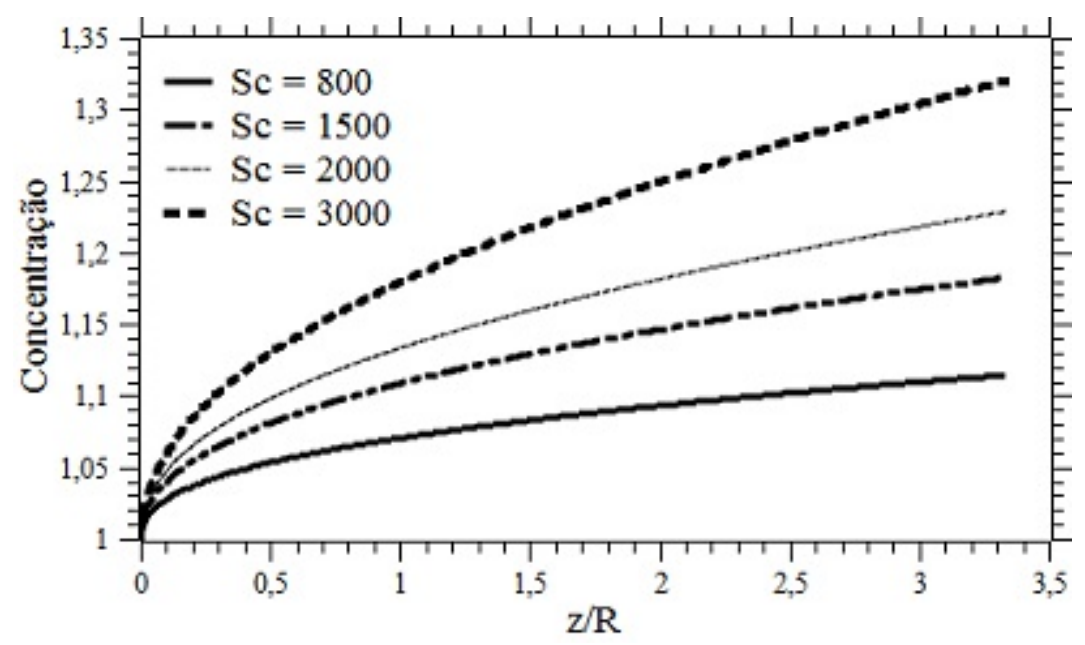

Figura 7: Concentração próxima à superfície permeável em função da coordenada axial, para diferentes número de Schmidt.

Na Fig. 7, tem-se os perfis de concentração próximos à superfície permeável em função da coordenada axial para diferentes números de Schmidt. Percebe-se que a concentração próxima à superfície permeável, ao longo do tubo, cresce a medida que o número de Schmidt aumenta, isso se deve ao fato que quando o número de Schmidt aumenta, o coeficiente de difusão diminui, 
consequentemente, espera-se que as partículas se acumulem perto da superfície permeável do tubo, o que concorda com a previsão da literatura [7, 8].

\section{Conclusões}

A investigação de um modelo matemático para o estudo do mecanismo de transferência de massa associado ao processo de filtração tangencial, apresentado nesse trabalho, revelou a influência da discretização dos termos convectivos nos resultados numéricos. Observou-se na Fig. 4 que dos dois esquemas convectivos testados, o esquema upwind de segunda ordem foi mais adequado nesse processo. Com esse esquema, verificou-se que os efeitos de parâmetros físicos, número de Reynolds e Schmidt (Figs. 6 e 7), nos perfis de concentração, em região próxima à superfície da membrana, possuem comportamento qualitativamente compatível com a tendência geral da literatura. Portanto, tem-se que o modelo numérico proposto se mostrou adequado para o problema físico estudado.

\section{Referências bibliográficas}

[1] RIPPERGER, S.; ALTMANN, J. Crossflow microfiltration: state of the art. Separation and Purification Technology, v. 26, n. 1, p. 19-31, 2002.

[2] ZEMAN, L. J.; ZYDNEY, A. L. Microfiltration and ultrafiltration: principles and applications. New York: Marcel Dekker, 1996.

[3] LEE, Y.; CLARK, M. M. A numerical model of steady-state permeate flux during cross-flow ultrafiltration. Desalination,v. 109, n. 3, p. 241-251, 1997.

[4] MIGNARD, D.; GLASS, D. H. Fouling during the cross-flow ultrafiltration of protein: a mass-transfer model. Journal of Membrane Science, v. 186, n. 1, p. 133-143, 2001.

[5] PARIS, J.; GUICHARDON, P.; CHARBIT, F. Transport phenomena in ultrafiltration: a new two-dimensional model compared with classical models. Journal of Membrane Science, v. 207, n. 1, p. 43-58, 2002.

[6] RICHARDSON, C. J.; NASSEHI, V. Finite element modelling of concentration profiles in flow domains with curved porous boundaries. Chemical Engineering Science, v. 58, n. 12, p. 2491-2503, 2003.

[7] DAMAK, K. et al. Concentration polarisation in tubular membranes: a numerical approach. Desalination, v. 171, n. 2, p. 139-153, 2004.

[8] VENEZUELA, A. L.; PÉREZ-GUERRERO, J. S.; FONTES, S. R. Hybrid modeling of convective laminar flow in a permeable tube associated with the cross-flow process. Communications in Nonlinear Science and Numerical Simulation, v. 14, n. 3, p. 795-810, 2009. 
[9] SILVA, J. M.; FERREIRA, V. G.; FONTES, S. R. An evaluation of three upwinding approximations for numerical modeling the flow in tubular membrane of Newtonian and non-Newtonian fluids. Applied Mathematics and Computation, v. 217, n. 20, p. 7955-7965, 2011.

[10] ALHUMAIZI, K. Comparison of finite difference methods for the numerical simulation of reacting fow. Computers \& Chemical Engeineering, v. 28, n. 9, p. 1759-1769, 2004.

[11] DARU, V.; TENAUD, C. Evaluation of tvd high resolution schemes for unsteady viscous shocked fows. Computers \& Fluids, v. 30, n. 1, p. 89-113, 2001.

[12] FORTUNA, A. O. Técnicas computacionais para dinâmica dos fluidos: conceitos básicos e aplicações. São Paulo: Editora da Universidade de São Paulo, 2000.

[13] WATERSON, N. P.; DECONINCK, H. Design principles for bounded higher-order convection schemes: a unified approach. Journal of Computational Physics, v. 224, n. 1, p. 182-207, 2007.

[14] PAES, E. M. T.; SILVA, J. M. Estudo numérico da concentração de partículas para escoamento em tubo permeável aplicado ao processo de filtração tangencial. In: ENCONTRO REGIONAL DE MATEMATICA APLICADA E COMPUTACIONAL, 5., 2018, Bauru. Caderno de trabalhos completos e resumos..., Bauru: Unesp, Faculdade de Ciências, 2018, p. 428-429. Disponível em: <http://www.fc.unesp.br/index.php\#!/departamentos/matematica/eventos2341/ermac2018/caderno-de-trabalhos-e-resumos/> . Acesso em: 5 ago. 2018.

[15] YUAN, S.W.; FUNKELSTEIN, A.B.; BROOKLYN, N.Y. Laminar pipe flow with injection and suction through a porous wall. Transactions of the ASME, v. 78, p. 719-724, 1956.

[16] GERALDES, V.; SEMIÃO, V.; PINHO, M. N. Flow and mass transfer modelling of nanofiltration. Journal Membrane Science, v. 191, n. 1-2, p. 109-128, 2001.

[17] FRANCO, N. B. Cálculo numérico. São Paulo: Pearson, 2007.

[18] MOREIRA, B. A. Obtenção de correlações para estimativa do coeficiente convectivo de transferência de massa para a geometria esférica a partir da técnica de sublimação do naftaleno. 2010. 64 f. Tese (Mestrado em Engenharia Química) - Faculdade de Engenharia Química, Universidade Federal de Uberlândia, Uberlândia, 2010. 